8. Shivaram PS, Girish KP. Alagille syndrome and the liver. Current insight. Euroasian J Hepatogastroenterol 2018; 8: 140-7.

9. Kamath BM, Schwarz KB, Hadžic N. Alagille syndrome and liver transplantation. J Paediatr Gastroenterol Nutr 2010; 50: 11-5.

10. Emerick KM, Rand EB, Goldmuntz E, Krantz ID, Spinner NB Piccoli DA. Features of Alagille syndrome in 92 patients: frequency and relation to prognosis. Hepatology 1999; 29: 822-9.

11. Drouin E, Russo P, Tuchweber B, Mitchell G, asquin-Weber A. North American Indian cirrhosis in children: a review of 30 cases. $J$ Pediat Gastroenterol Nutr 2000; 31: 395-404.

12. Davit-Spraul A, Gonzales E, Baussan C, Jacquemin. The spectrum of liver diseases related to ABCB4 gene mutations: pathophysiology and clinical aspects. Semin Liver Dis 2010; 30: 134-46.

DOI: https://doi.org/10.1016/j.pathol.2020.04.015

\section{Ewing sarcoma of urinary bladder occurring simultaneously with high grade papillary urothelial carcinoma}

Sir,

Ewing sarcoma (ES) is a highly aggressive small round cell malignant tumour, most often occurring in the bone of children and adolescents. ES can also occur in extraskeletal organs, such as the liver, testis, uterus, kidney, ovary, parotid gland, lung, pancreas, urinary bladder, rectum, heart, gall bladder and cervix. ${ }^{1,2}$ ES occurring in the urinary bladder is very rare, and only 18 cases have been reported in the literature to date. ${ }^{1,3-11}$ Here we report another case of primary ES in the urinary bladder of a 78-year-old woman. It is the first reported case of ES occurring simultaneously with high grade papillary urothelial carcinoma (UC).

A 78-year-old woman presented with painless gross haematuria for 2 months, which became progressively worse, with blood clot, urinary frequency and urgency. Computed tomography urography (CTU) demonstrated a $63 \times 44 \mathrm{~mm}$ mass from the right posterior bladder wall, with hydroureter and hydronephrosis (Fig. 1A). Contrast-enhanced CT of the pelvic cavity revealed a $62 \times 42 \mathrm{~mm}$, lobulated, abnormally diffused enhancement, narrow-based pedunculated mass arising from the bladder wall, which was extending into the right uretero-bladder junction, suggesting UC. No distant organ or lymph node metastases were detected. Complete transurethral resection of the tumour was performed. Radical cystectomy was not performed. The patient died 2 months after transurethral resection, without chemotherapy and radiotherapy.

Microscopic examination showed high grade malignant neoplasm with papillary features of the surface urothelium and small, round, blue cell tumour diffusely infiltrating the lamina propria without infiltration of muscularis propria (Fig. 1B). In high grade lesion, the papillae fused and had marked nuclear dysplasia. The tumour cells displayed cellular disorder, nuclear size variation, irregular and pleomorphic nuclei, and prominent nucleoli (Fig. 1C). Numerous mitoses could be seen. In situ intraepithelial neoplasia was identified. The diffusely infiltrating area of the lamina propria was composed of small round blue cells with scant cytoplasm, monotonous round to oval nuclei, fine granular chromatin and small nucleoli (Fig. 1D). The tumour cells were arranged in solid sheets with a prominent capillary network and necrosis. Rosette formation was not evident. The mitotic activity was abundant. On immunohistochemistry, the tumour cells infiltrating the lamina propria showed strong membranous CD99 (Fig. 2A), weak nuclear Fli-1 and focal synaptophysin expression. Pan-cytokeratin, EMA, CK7, CK20 (Fig. 2B), P40, p63, chromogranin, S100, melanoma (HMB45), Melan-A, desmin, smooth muscle actin, MyoD1, CD20, CD3, CD10, PAX5, CD30, MPO, bcl-6, MUM-1, SALL4, OCT3/4 and D2-40 were negative in the small round blue cells. The tumour cells of the surface papillary projections were positive for pan-cytokeratin, EMA, CK7, CK20, P40 and p63; the other markers were negative. GATA-3 was diffusely strong positive in the surface papillary projections and weak positive in some areas of the small round blue cells (Fig. 2C). The histomorphological findings and immunohistochemical profile were found to be consistent with ES simultaneously with high grade papillary UC.

Fluorescence in situ hybridisation (FISH) was carried out on formalin-fixed paraffin-embedded tumour sample and using the dual-colour break-apart probes for EWSR1. FISH analysis displayed a 22q12 translocation pattern for the EWSRl gene, thus confirming the diagnosis of ES (Fig. 2D)

ES is the second most common sarcoma of bone in children and young adults; nearly $80 \%$ of patients are younger than 20 years. Most cases are composed of uniform small round cells with round nuclei containing fine chromation and scanty clear or eosinophilic cytoplasm, exhibiting a variable degree of neuroectodermal differentiation. ES is characterised by recurrent balanced translocation involving the EWSRl gene on chromosome 22 and a member of the ETS family of transcription factors. ES of the urinary bladder is a rare entity and only 18 cases have been reported in the literature to date. Here, we presented the first reported case of ES of the bladder occurring simultaneously with high grade papillary UC.

The age of patients with primary ES in the bladder reported in the literature ranged from 10 to 81 years, with a mean age of 42 years. It occurred in a relatively older age group, with only four patients $(22.2 \%)$ younger than 20 years. Of the 18 patients, 10 were females and 8 were males, which showed a slight female predominance, with a sex ratio of 1.2:1. Haematuria was the most frequent presenting symptom; other reported presenting symptoms included dysuria, oliguria, urinary frequency, hydronephrosis, renal failure, oedema in lower extremities, pelvic and abdominal pain. The tumour size ranged from 2.0 to $15 \mathrm{~cm}$. Five cases were metastatic at the time of diagnosis and three tumours had perivesical infiltration. Radical cystectomy was performed in five patients and partial resection in another four patients; the remaining patients were managed with transurethral resection or conservative treatment.

Histologically, ES of the urinary bladder appear as sheets of uniform small round cells with round nuclei. The diagnosis is difficult as it is extremely rare in this location. ES must be distinguished from poorly differentiated UC, especially in the present case, because the treatment protocols are different. Strong membranous positivity of CD99, nuclear positivity of Fli-1 and absence of cytokeratin staining favours the diagnosis of ES. Other differential diagnoses include small cell carcinoma, lymphoma, rhabdomyosarcoma, small cell variant of malignant melanoma and synovial sarcoma. These diagnoses can be ruled out in most cases by immunohistochemistry. Absence of neuroendocrine 

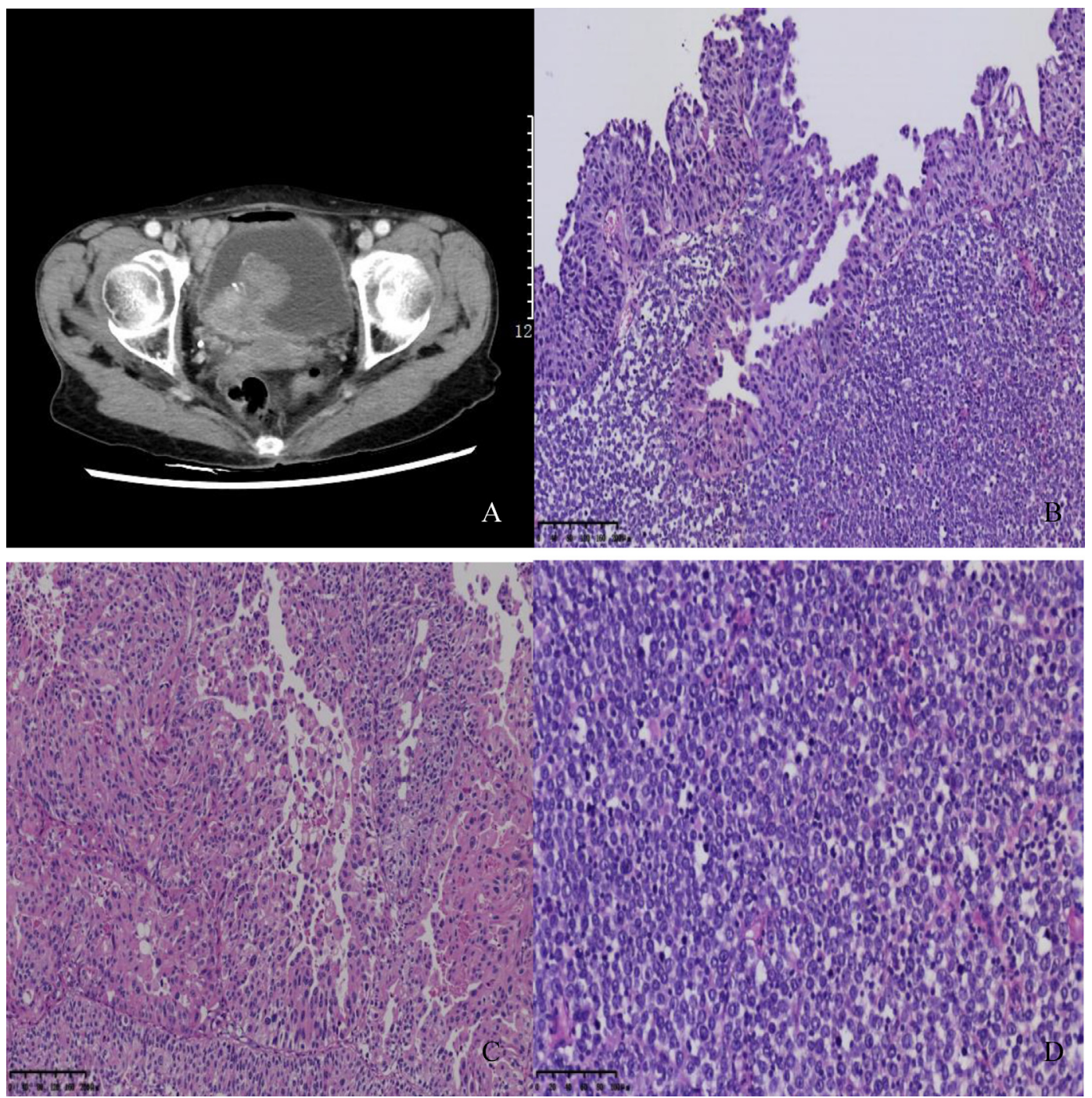

Fig. 1 (A) CTU revealed a large mass from the right posterior bladder wall. (B) Small blue round cell neoplasm underlying the surface urothelial carcinoma and infiltrating the lamina propria. (C) The area of urothelial carcinoma, with fused papillae and marked nuclear dysplasia in the tumour cells. (D) The area of Ewing sarcoma showing uniform tumour cells with round to oval nuclei and small nucleoli.

markers (synaptophysin and chromogranin) and cytokeratin staining excluded small cell carcinoma. Immunonegativity for CD20, CD3, CD10, PAX5, CD30, MPO, bcl-6 and MUM-1 ruled out lymphoma. Absence of melanocytic markers (S100, HMB45 and Melan-A) ruled out malignant melanoma. CD99 positivity can be seen in synovial sarcoma and rhabdomyosarcoma. Immunonegativity for desmin, smooth muscle actin and MyoD1 ruled out the possibility of rhabdomyosarcoma. Molecular analysis aids in the correct diagnosis between ES and synovial sarcoma. Synovial sarcoma is characterised by the $\mathrm{t}(\mathrm{X} ; 18)(\mathrm{p} 11 ; \mathrm{q} 11)$ translocation, leading to formation of a SS18-SSX fusion gene. ES show the EWS gene rearrangement by FISH and approximately $85 \%$ of cases harbour the reciprocal translocation $\mathrm{t}(11 ; 22)$ (q24;q12) and EWS/FLI-1 fusion transcript. A combination of immunohistochemical studies and molecular studies aids in the correct diagnosis. GATA-3 is a sensitive and specific marker for UC. Interestingly, GATA-3 was weakly expressed in some areas of ES in our case, which is an interesting phenomenon. This may be explained by the theory of the histogenesis of sarcomatoid carcinoma of the bladder. UC has a remarkable propensity for divergent differentiation. As noted previously, sarcomatoid carcinoma is typically a biphasic tumour with morphological and immunohistochemical evidence of both epithelial and mesenchymal differentiation, such as chondrosarcoma, leiomyosarcoma, osteosarcoma, or rhabdomyosarcoma. Molecular studies support the concept of monoclonal origin from urothelial stem cells with divergent differentiation. ${ }^{12}$

Primary ES of the urinary bladder is a rare entity. Definitive guidelines regarding its management and treatment have not been established. A multimodal approach is mandatory for the management of ES. Radical resection, adjuvant radiation and chemotherapy are recommended. Recently, neoadjuvant chemotherapy followed by surgical resection was effective. ${ }^{10,11}$ Our patient simultaneously had high grade papillary UC, so discussion of the management and treatment was necessary. Unfortunately, the patient did not receive any treatment after surgery because she was in poor condition. The prognosis of ES of the bladder is poor; the survival time reported in the literature ranges from 2 weeks to more than 36 months, and elderly patients have lower survival rates. Our 

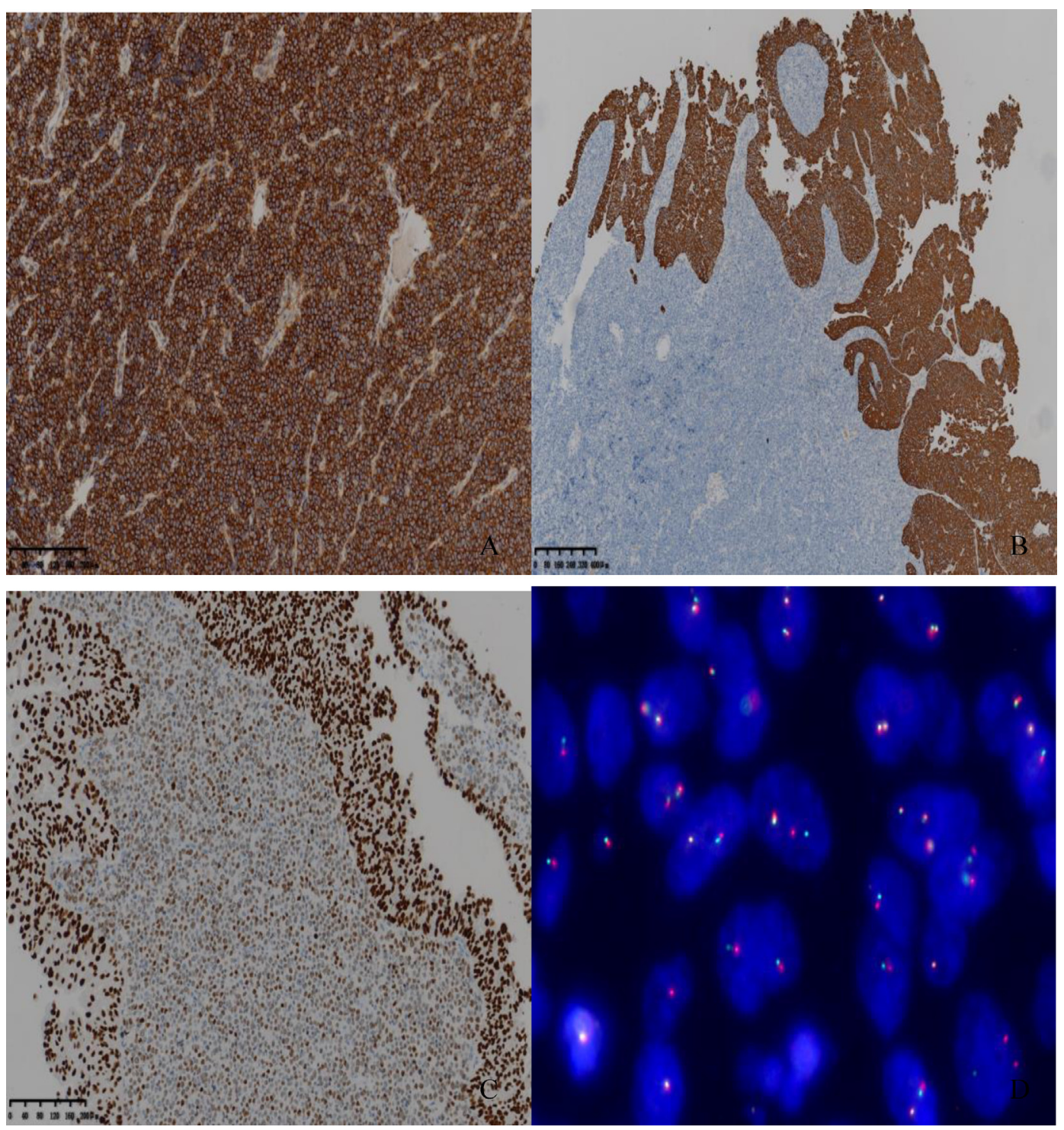

Fig. 2 (A) Diffuse membranous positive immunohistochemical stain for CD99 in Ewing sarcoma. (B) The surface urothelial carcinoma showed diffusely positive CK20 expression, while Ewing sarcoma in the lamina propria was negative. (C) GATA-3 was diffusely strong positive in the surface urothelial carcinoma and weak positive in Ewing sarcoma. (D) FISH analysis showing a rearrangement of the EWSRl gene (22q12).

patient was an older woman and died 2 months after the surgery.

In conclusion, ES of the urinary bladder is very rare and this entity is likely under-recognised given its rarity. Awareness of this entity is essential for an accurate diagnosis and thus appropriate treatment. The diagnosis of ES requires a combination of histological, immunohistochemical and molecular analysis. The present case is an interesting case because ES occurred simultaneously with UC. The description of more new cases is necessary to improve our knowledge and experience.

Conflicts of interest and sources of funding: The authors state that there are no conflicts of interest to disclose.

\section{Hui-Zhi Zhang, Su-Ying Wang, Xiao-Han Shen}

Department of Pathology, Ningbo Diagnostic Pathology Center, Ningbo, Zhejiang, China
Contact Hui-Zhi Zhang.

E-mail: zhanghuizhiyang@163.com

1. Tonyalı Ş, Yazıcı S, Yeşilırmak A, et al. The Ewing's sarcoma family of tumors of urinary bladder: a case report and review of the literature. Balkan Med J 2016; 33: 462-6.

2. Zhang Y, Nong W, Ren Y, et al. Ewing's sarcoma of the cervix: a case report and review of literature. Histol Histopathol 2019; 5: 18181.

3. Al Meshaan MK, Nayef M, Kwaider T, et al. Peripheral primitive neuroectodermal tumor of the urinary bladder in an Arab woman with history of squamous cell carcinoma: a case report. J Med Case Rep 2009; 3: 6840 .

4. Busato Jr WF, Almeida GL, Ogata DC. Primary primitive neuroectodermal tumor of the bladder: histologic and clinical features of 9 cases. Clin Genitourin Cancer 2011; 9: 63-7.

5. Rao RN, Sinha S, Babu S, et al. Fine-needle aspiration cytology of primitive neuroectodermal tumor of the urinary bladder: a case report. Diagn Cytopathol 2011; 39: 924-6.

6. Zheng Y, Tan F, Wang L, et al. Primary primitive neuroectodermal tumor of the urinary bladder: a case report and literature review. Med Oncol 2011; 28 (Suppl 1): S388-91.

7. Okada Y, Kamata S, Akashi T, et al. Primitive neuroectodermal tumor/ Ewing's sarcoma of the urinary bladder: a case report and its molecula diagnosis. Int J Clin Oncol 2011; 16: 435-8. 
8. Sueyoshi R, Okawada M, Fujimura J, et al. Successful complete resection of Ewing sarcoma arising from the bladder in a 10-year-old boy after chemotherapy. Pediatr Surg Int 2014; 30: 965-9.

9. Osone S, Hosoi H, Tanaka K, et al. A case of a Ewing sarcoma family tumor in the urinary bladder after treatment for acute lymphoblastic leukemia. J Pediatr Hematol Oncol 2007; 29: 841-4.

10. Lam CJ, Shayegan B. Complete resection of a primitive neuroectodermal tumour arising in the bladder of a 31-year-old female after neoadjuvant chemotherapy. Can Urol Assoc J 2016; 10: E264-7.
11. Vallonthaiel AG, Kaur K, Jain D, et al. Ewing sarcoma of urinary bladder showing EWSR1 rearrangement on FISH analysis an unique response to chemotherapy. Clin Genitourin Cancer 2016; 14 e183-6.

12. Cheng L, Zhang SB, Alexander R, et al. Sarcomatoid carcinoma of the urinary bladder: the final common pathway of urothelial carcinoma dedifferentiation. Am J Surg Pathol 2011; 35: e34-46.

DOI: https://doi.org/10.1016/j.pathol.2020.05.004 